\title{
Associations between meal and snack frequency and overweight and abdominal obesity in US children and adolescents from National Health and Nutrition Examination Survey (NHANES) 2003-2012
}

\author{
Kentaro Murakami $^{1 *}$ and M. Barbara E. Livingstone ${ }^{2}$ \\ ${ }^{1}$ Department of Nutrition, School of Human Cultures, University of Shiga Prefecture, Shiga 522 8533, Japan \\ ${ }^{2}$ Northern Ireland Centre for Food and Health, Ulster University, Coleraine BT52 1SA, UK \\ (Submitted 14 September 2015 - Final revision received 7 January 2016 - Accepted 10 February 2016 - First published online 22 March 2016)
}

\section{Abstract}

The association between eating frequency (EF) and adiposity in young populations is inconsistent. This cross-sectional study examined associations of EF, meal frequency (MF) and snack frequency (SF) with adiposity measures in US children aged 6-11 years ( $n$ 4346) and adolescents aged 12-19 years ( $n$ 6338) participating in the National Health and Nutrition Examination Survey 2003-2012. Using data from two 24-h dietary recalls, all eating occasions providing $\geq 210 \mathrm{~kJ}$ of energy were divided into meals or snacks based on contribution to energy intake ( $\geq 15$ or $<15 \%$ ), self-report and time (06.00-09.00, 12.00-14.00 and 17.00-20.00 hours or others). When analysed without adjustment for the ratio of reported energy intake:estimated energy requirement (EI:EER), all measures of EF, MF and SF showed inverse or null associations with overweight (BMI $\geq 85$ th percentile of BMI-for-age) and abdominal obesity (waist circumference $\geq 90$ th percentile) in both children and adolescents. After adjustment for EI:EER, however, EF and SF, but not MF, showed positive associations in children, irrespective of the definition of meals and snacks. In adolescents, after adjustment for EI:EER, positive associations were observed for EF (abdominal obesity only), SF based on energy contribution and MF based on self-report, whereas there was an inverse association between MF based on energy contribution and overweight. In conclusion, higher SF and EF, but not MF, were associated with higher risks of overweight and abdominal obesity in children, whereas associations varied in adolescents, depending on the definition of meals and snacks. Prospective studies are needed to establish the associations observed here.

\section{Key words: Meal frequency: Snack frequency: Obesity: National Health and Nutrition Examination Survey}

Overweight and obesity in children and adolescents continue to be major public health concerns in many countries including the $\mathrm{USA}^{(1,2)}$. Excess body fatness has been shown to adversely affect children and adolescents by reducing their health-related quality of life $\mathrm{e}^{(3,4)}$, and is also associated with several risk factors for later chronic diseases ${ }^{(5,6)}$. As established body fatness is difficult to treat successfully, investigation of modifiable lifestyle factors that influence body fatness in children and adolescents is a high public health priority.

A large number of epidemiological studies conducted among young populations have shown an inverse relationship between eating frequency $(\mathrm{EF})$ - that is, the number of times per day a person eats, regardless of whether that eating occasion is designated as a meal or snack - and adiposity measures $^{(7-20)}$, with some exceptions ${ }^{(21-26)}$. However, the results should be interpreted cautiously with regard to methodological limitations. First, the assessment of EF has often relied on a series of self-report questions ${ }^{(9,10,13-17,19,23)}$. Only a few studies have assessed EF on the basis of information on actual dietary behaviours (using dietary record or 24-h recall) ${ }^{(7,18,21,26)}$.

In addition, the relationships of $\mathrm{EF}$ with adiposity measures may be confounded by possible under-reporting of $\mathrm{EF}$ concomitant with the under-reporting of energy intake (EI) by obese or overweight subjects ${ }^{(27,28)}$. For example, one study found that when subjects with implausible EI were eliminated from the analytic sample, the inverse relation between $\mathrm{EF}$ and BMI percentile no longer existed ${ }^{(25)}$. Further, interpreting the literature on $\mathrm{EF}$ is complicated by the fact that there is no consensus about what constitutes a snack, a meal or an eating occasion. Although some researchers have relied on respondents' self-identification of meals, snacks or eating occasions $^{(8-10,13-19,23,24)}$, others have attempted to use more objective criteria (based on clock time, energy content/ contribution or both) ${ }^{(7,20-22,25,26)}$. As a consequence of these

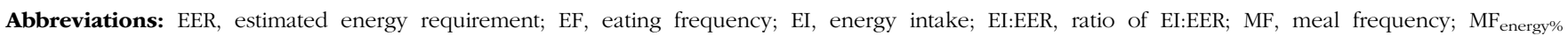

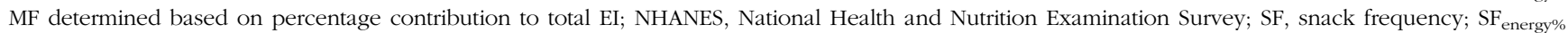
SF determined based on percentage contribution to total EI; WC, waist circumference.

* Corresponding author: Dr K. Murakami, fax +81 74949 8499, email kenmrkm@m.u-tokyo.ac.jp 
methodological limitations, more robust data analyses are needed to resolve this issue.

More importantly, potentially different effects of meal frequency (MF) and snack frequency (SF) have not been investigated simultaneously using different definitions of meals and snacks. An accurate distinction between meals and snacks is of importance, because they are hypothesised to have opposite effects on energy balance. A high MF may prevent fat mass deposition, whereas snacking may contribute to fat mass deposition $^{(29)}$. This is also important for the development of science-based recommendations of meal and snack patterns for consumers ${ }^{(30)}$.

The aim of the present cross-sectional study in a representative sample of US children and adolescents based on the data from the National Health and Nutrition Examination Survey (NHANES) was to examine the relationship of $\mathrm{EF}, \mathrm{MF}$ and $\mathrm{SF}$ with overweight and abdominal obesity, by focusing on the confounding of EI misreporting and the use of different definitions of eating occasions, meals and snacks.

\section{Methods}

\section{Survey design and analytical sample}

The present cross-sectional analysis was based on public domain data from NHANES, a continuing population-based survey that uses a complex, stratified, multistage probability sample design to create a representative sample of the non-institutionalised civilian US population ${ }^{(31,32)}$. The survey examines about 5000 persons each year, and data are released in 2-year cycles. The unweighted response rates for the examined persons aged 6-19 years for NHANES 2003-2004, 2005-2006, 2007-2008, 2009-2010 and 2011-2012 were 84, 83, 83,86 and $77 \%$, respectively ${ }^{(33)}$. The NHANES protocol was approved by National Center for Health Statistics Research Ethics Review Board, and written informed consent was obtained from all participants or proxies. The documentation and data used for each of these surveys were downloaded from the NHANES website ${ }^{(34)}$.

For the present analysis, five survey cycles (i.e. 2003-2004, 2005-2006, 2007-2008, 2009-2010 and 2011-2012) were combined to produce estimates with greater precision and smaller sampling error. The analytical sample was limited to children and adolescents aged 6-19 years with data from two complete, reliable and self-reported 24-h dietary recalls, determined by the National Center for Health Statistics ( $n$ 11719; the time interval between the two 24-h recalls was 3-10d). After excluding pregnant ( $n$ 96) and lactating ( $n$ 15) respondents, as well as those with missing information on the variables of interest ( $n$ 924), the final analytic sample included 10684 respondents from NHANES 2003-2012.

\section{Anthropometric measurements}

Body weight and height were measured by trained interviewers using standardised procedures with calibrated equipment. Waist circumference (WC) was measured by trained personnel with a soft tape placed horizontally just above the iliac crest at minimal respiration. BMI $\left(\mathrm{kg} / \mathrm{m}^{2}\right)$ was calculated as weight $(\mathrm{kg})$ divided by height squared $\left(\mathrm{m}^{2}\right)$. The percentile of BMI for age was calculated using SAS programme for growth charts available from the Centers for Disease Control and Prevention ${ }^{(35,36)}$. Overweight (including obesity) was defined as $\mathrm{BMI} \geq 85$ th percentile of BMI for age. Abdominal obesity was defined as WC equal or above the sex- and age-specific 90th percentile based on data from NHANES III $(1988-1994)^{(37)}$.

\section{Dietary assessment}

All surveys collected dietary information using two 24-h dietary recalls. The first recall was conducted by face-to-face interviews, with the second recall being conducted by telephone 3-10 d after the first recall on a different day of the week (but not necessarily, e.g., 1 weekday and 1 weekend day). The dietary data were collected using an automated five-step multiple-pass approach - namely, the US Department of Agriculture Automated Multiple-Pass Method ${ }^{(34,38-41)}$. Participants were asked to report the time each food and beverage was consumed and to classify each eating occasion from a pre-defined list of categories, which were used to define meals and snacks, as described later. Proxies, most commonly a parent, assisted with the dietary interview of children aged 6-11 years; dietary intake was self-reported by adolescents aged 12-19 years. Estimates of intakes of energy and selected nutrients from all reported foods and beverages were calculated using the US Department of Agriculture Food and Nutrient Database for Dietary Studies ${ }^{(34)}$. Values of nutrient intake were energy-adjusted using the density method (i.e. percentage of energy for energy-providing nutrients and amount per $4184 \mathrm{~kJ}$ of energy for dietary fibre).

\section{Definitions of eating frequency, meal frequency and snack frequency}

Data from the two 24-h dietary recalls were also used to calculate the mean number of eating occasions per day - that is, EF. Eating occasions were defined as any occasion when any food or drink was consumed ${ }^{(7,12,20,22,26,42-44)}$. In many previous studies, if two eating occasions occurred in $\leq 15 \mathrm{~min}$, both events were counted as a single eating occasion; when $>15$ min separated two eating occasions, they were considered distinct eating occasions ${ }^{(20,21,26,44,45)}$. In the present study, however, all foods and beverages reported at one discrete clock time were considered as part of one eating occasion, because almost all eating episodes (>99.5\%) occurred at least $15 \mathrm{~min}$ apart in NHANES $^{(46)}$. EF was calculated based on all eating occasions except for those providing $<210 \mathrm{~kJ}$ of energy. This calculation method has been used in several previous studies ${ }^{(26,42-45,47)}$ and was chosen to avoid giving undue weight to eating occasions that only included water, low-energy beverages or small quantity of foods. Use of differently calculated EF, that is, EF based on all eating occasions or EF based on all eating occasions except for those providing no energy, did not change the results (data not shown).

All eating occasions were divided into either meals or snacks using three different published definitions: on the basis of 
(1) contribution to total $\mathrm{EI}^{(7)}$, (2) self-reported name of eating occasion $^{(46)}$ and (3) clock time ${ }^{(22)}$. For the first definition ${ }^{(7)}$, a meal was defined as any eating episode comprising $\geq 15 \%$ of total EI, regardless of the time of the day or composition of foods or beverages consumed. All other eating episodes were classified as a snack. For each participant, MF and SF determined based on percentage contribution to total EI were thus calculated (hereafter referred to as $\mathrm{MF}_{\text {energy } \%}$ and $\mathrm{SF}_{\text {energy\%, }}$ respectively). For the second definition ${ }^{(46)}$, eating occasions with self-reported names such as 'breakfast', 'brunch', 'lunch', 'supper' and 'dinner' or their equivalents in Spanish were considered meals. All other self-reported eating events were considered as snacks. For each participant, MF and SF determined based on self-report were thus calculated. For the third definition ${ }^{(22)}$, meals were defined as eating events reported during select times of the day, that is, 06.00-09.00, 12.00-14.00 and 17.00-20.00 hours. All other eating occasions were considered snacks. For each participant, MF and SF determined based on the time consumed were thus calculated.

\section{Assessment of non-dietary variables}

Race/ethnicity was categorised as non-Hispanic white, nonHispanic black, Mexican-American and others. As an indicator of socio-economic status, family poverty income ratio was considered ( $<130,130-349$ and $\geq 350 \%$ ). The physical activity variable was created as follows: for children aged 6-11 years, the responses to the question on the number of times per week that play or exercise was hard enough to induce sweat in the past 7 d (NHANES 2009-2012) or without a specified period (NHANES 2003-2008) were as follows - 0-3 times=low, 4-6 times $=$ moderate and 7 times $=$ active; for adolescents aged 12-19 years, the responses to two different questions on any leisure time moderate or vigorous activities lasting $\geq 10 \mathrm{~min}$ in the past 30d (NHANES 2003-2006) or without a specified period (NHANES 2007-2012) were as follows - no to both the questions $=$ low, yes to one question $=$ moderate and yes to both two questions = active. The hours of screen time were determined from questions on television/video watching $(\mathrm{h} / \mathrm{d})$ or computer use $(\mathrm{h} / \mathrm{d})$ over the past $30 \mathrm{~d}$ (except for adolescents aged 12-19 years in NHANES 2007-2008 and 2009-2010 for which information on sedentary activity was used), which were categorised as $<2, \geq 2$ to $<4, \geq 4$ to $<6$ and $\geq 6 \mathrm{~h} / \mathrm{d}$.

\section{Evaluation of energy intake reporting}

Misreporting of EI was evaluated based on the ratio of EI: estimated energy requirement (EER). EER was calculated using sex-, age- and weight status-specific equations from the US Dietary Reference Intakes, based on sex, age, body height and weight, and physical activity ${ }^{(48)}$. Owing to a lack of an objective measure of physical activity in the present study, 'low active' level of physical activity (i.e. physical activity level $\geq 1.4$ to $<1 \cdot 6)^{(48)}$ was assumed for all subjects during this calculation, based on accelerometer data from NHANES 2003-2006 ${ }^{(49,50)}$.

\section{Statistical analysis}

Statistical analyses were performed for children aged 6-11 years ( $n$ 4346) and adolescents aged $12-19$ years ( $n$ 6338) separately, using SAS statistical software (version 9.2, SAS Institute). The mean of the $2 \mathrm{~d}$ of dietary recalls was used for all dietary variables, rather than calculating usual intake, because, although statistical methods for estimating usual dietary intake are based on the assumption that the dietary assessment method (24-h dietary recall in this case) is an unbiased instrument for measuring food intake ${ }^{(51)}$, this seems violated in many cases at least for EF variables ${ }^{(25-28)}$. This approach has been used in several previous studies ${ }^{(52,53)}$. All the analyses used the NHANES-provided sampling weights that were calculated to take into account unequal probabilities of selection resulting from the sample design, non-response and planned over-sampling of selected subgroups, so that the results are representative of the US community-dwelling population $^{(32,54)}$. For $\mathrm{EF}, \mathrm{MF}$ and $\mathrm{SF}$, sample-weighted means (with SE) were generated using PROC SURVEYMEANS procedure. Differences in these variables across categories of each of the characteristics were examined by Wald's $F$ test using PROC SURVEYREG procedure. Associations of EF, MF, SF and adiposity measures with EI and EI:EER were investigated by linear regression analyses using PROC SURVEYREG procedure. Linear regression and logistic regression analyses were performed to explore the associations of $\mathrm{EF}, \mathrm{MF}$ and $\mathrm{SF}$ (independent variables) with measures of fatness (dependent variables). EF, MF and SF were analysed continuously after confirming the linearity of relations using tertile, quartile and quintile categories. Using the PROC SURVEYREG procedure, the adjusted regression coefficients (with $\mathrm{SE}$ ) of variation of $\mathrm{BMI}$ percentile and $\mathrm{WC}$ by one increase in $\mathrm{EF}, \mathrm{MF}$ and $\mathrm{SF}$ were calculated. In addition, using the PROC SURVEYLOGISTIC procedure, OR and 95\% CI for overweight and abdominal obesity per one increase in $\mathrm{EF}, \mathrm{MF}$ and $\mathrm{SF}$ were calculated. The potential confounding factors considered (in model 1) were sex, age, race/ethnicity, family poverty income ratio, physical activity, watching television and computer use, survey cycle, protein intake, fat intake, total sugar intake and dietary fibre intake. EI:EER was further included as a potential confounding factor (in model 2). All reported $P$ values are two-tailed, and $P<0.05$ was considered to be statistically significant.

\section{Results}

Dietary characteristics and adiposity measures of the subjects are presented in Table 1. EF, MF and SF were higher in children than in adolescents, irrespective of the definition applied. When compared with EER, EI was on average over-reported by $6 \%$ in children but under-reported by $14 \%$ in adolescents. There was no difference in the prevalence of overweight and abdominal obesity between children and adolescents. The correlations among three measures of MF were relatively weak in both children (Pearson's $r$ : 0.09-0.34) and adolescents (Pearson's $r$ : 0.24-0.40), whereas there were relatively strong correlations among three measures of $\mathrm{SF}$ in both children 
Table 1. Dietary characteristics and adiposity measures of the subjects: National Health and Nutrition Examination Survey (NHANES) 2003-2012* (Mean values with their standard errors)

\begin{tabular}{|c|c|c|c|c|c|}
\hline & \multicolumn{2}{|c|}{ Children aged $6-11$ years ( $n$ 4346) } & \multicolumn{2}{|c|}{ Adolescents aged $12-19$ years ( $n$ 6338) } & \multirow[b]{2}{*}{$P \dagger$} \\
\hline & Mean & $\mathrm{SE}$ & Mean & $\mathrm{SE}$ & \\
\hline EF (times/d)‡ & 4.76 & 0.03 & 4.13 & 0.03 & $<0.0001$ \\
\hline $\mathrm{MF}_{\text {energy\% }}($ times $/ \mathrm{d}) \ddagger \S$ & $2 \cdot 88$ & 0.01 & $2 \cdot 68$ & 0.01 & $<0.0001$ \\
\hline $\mathrm{SF}_{\text {energy\% }}($ times $/ \mathrm{d}) \ddagger \S$ & 1.88 & 0.03 & 1.45 & 0.02 & $<0.0001$ \\
\hline $\mathrm{MF}_{\text {self-report }}($ times $/ \mathrm{d}) \ddagger \|$ & 2.88 & 0.01 & 2.55 & 0.01 & $<0.0001$ \\
\hline $\mathrm{SF}_{\text {self-report }}($ times $/ \mathrm{d}) \ddagger \|$ & 1.88 & 0.03 & 1.59 & 0.03 & $<0.0001$ \\
\hline $\mathrm{MF}_{\text {time }}($ times $/ \mathrm{d}) \pm \boldsymbol{\uparrow}$ & 2.96 & 0.02 & $2 \cdot 40$ & 0.02 & $<0.0001$ \\
\hline $\mathrm{SF}_{\text {time }}($ times $/ \mathrm{d}) \neq \boldsymbol{I}$ & 1.80 & 0.02 & 1.73 & 0.03 & 0.046 \\
\hline $\mathrm{EI}(\mathrm{kJ} / \mathrm{d})$ & 8201 & 67 & 8987 & 88 & $<0.0001$ \\
\hline El:EER & 1.06 & 0.01 & 0.86 & 0.01 & $<0.0001$ \\
\hline Protein intake ( $\%$ of energy) & 14.2 & 0.1 & 14.9 & 0.1 & $<0.0001$ \\
\hline Fat intake ( $\%$ of energy) & 33.1 & 0.2 & 33.3 & 0.2 & 0.46 \\
\hline Carbohydrate intake ( $\%$ of energy) & 53.9 & 0.2 & $52 \cdot 5$ & 0.2 & $<0.0001$ \\
\hline Total sugar intake (\% of energy) & 26.5 & 0.2 & $25 \cdot 1$ & 0.2 & $<0.0001$ \\
\hline Dietary fibre intake $(\mathrm{g} / 4184 \mathrm{~kJ})$ & 7.1 & 0.1 & $6 \cdot 7$ & 0.1 & $<0.0001$ \\
\hline BMI percentile & $62 \cdot 8$ & 0.8 & $64 \cdot 4$ & 0.8 & 0.20 \\
\hline Waist circumference $(\mathrm{cm})$ & 64.8 & 0.4 & $81 \cdot 8$ & 0.4 & $<0.0001$ \\
\hline Overweight $(\%)^{\star \star}$ & 33.4 & 1.5 & 33.9 & $1 \cdot 1$ & 0.77 \\
\hline Abdominal obesity (\%)†† & $17 \cdot 1$ & $1 \cdot 1$ & $19 \cdot 5$ & 0.9 & 0.08 \\
\hline
\end{tabular}

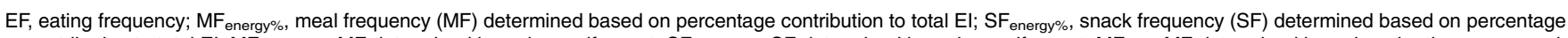
contribution to total El; $\mathrm{MF}_{\text {self-report, }} \mathrm{MF}$ determined based on self-report; $\mathrm{SF}_{\text {self-report, }} \mathrm{SF}$ determined based on self-report; MF $\mathrm{SF}_{\text {time, }}$ SF determined based on the time consumed; El, energy intake; El:EER, ratio of El:estimated energy requirement.

* All mean values are weighted to reflect the survey design characteristics. Analyses are based on subjects with complete data on two 24-h dietary recalls as well as complete information on the variables of interest. All dietary variables are based on average values of the two 24-h dietary recalls.

$\dagger P$ values for differences between children and adolescents based on independent $t$ test.

$\ddagger$ On the basis of all occasions except for those providing $<210 \mathrm{~kJ}$ of energy.

$\S \mathrm{A}$ meal was defined as any eating episode comprising $\geq 15 \%$ of total El, regardless of the time of the day or composition of foods and beverages consumed; all other eating episodes were classified as snacks.

॥ Self-reports of breakfast, brunch, lunch, supper and dinner or their equivalents in Spanish were considered as meals; all other self-reported eating events were considered as snacks.

TI Meals were defined as eating events reported during select times of the day (06.00-09.00, 12.00-14.00 and 17.00-20.00 hours); all other eating occasions were considered as snacks.

** Including obesity. Defined as BMI $\geq 85$ th percentile of BMl for age according to growth charts available from the Centers for Disease Control and Prevention ${ }^{(35,36)}$.

t† Defined as waist circumference equal or above the sex- and age-specific 90th percentile based on data from NHANES III (1988-1994) ${ }^{(37)}$.

(Pearson's r: 0.55-0.81) and adolescents (Pearson's r: 0.55-0.79) (online Supplementary Table S1). All measures of MF and $\mathrm{SF}$ were positively correlated with $\mathrm{EF}$, although the strength of the correlations varied (Pearson's $r$ ranging from $0 \cdot 13$ ( $\mathrm{MF}_{\text {energy } \%}$ in children) to 0.89 (SF based on self-report in children)).

\section{Associations of eating frequency, meal frequency and snack frequency with subjects characteristics}

Table 2 shows $\mathrm{EF}, \mathrm{MF}_{\text {energy\% }}$ and $\mathrm{SF}_{\text {energy\% (as examples) }}$ according to categories of subject characteristics. Although EF was higher in boys in both age groups, $\mathrm{MF}_{\text {energy\% }}$ was higher in male

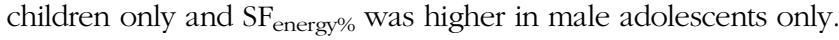

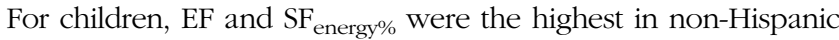
whites and the lowest in non-Hispanic blacks. For adolescents, EF and $\mathrm{SF}_{\text {energy\% }}$ were the highest in others, whereas $\mathrm{MF}_{\text {energy\% }}$ was the highest in non-Hispanic whites, with the lowest values in non-Hispanic blacks. There were positive associations between family poverty income ratio and $\mathrm{EF}$ (adolescents only) and $\mathrm{SF}_{\mathrm{e}}$ nergy\%. All $\mathrm{EF}, \mathrm{MF}_{\text {energy\% }}$ and $\mathrm{SF}_{\text {energy\% }}$ values were positively associated with physical activity in adolescents only. There were inverse associations between watching television and computer use and $\mathrm{EF}$ and $\mathrm{SF}_{\text {energy\% }}$ in adolescents only. For survey cycle, only $\mathrm{SF}_{\text {energy } \%}$ differed in adolescents, with the highest in 2003-2004 and the lowest in 2007-2008.
Associations of eating frequency, meal frequency, snack frequency and adiposity measures with energy intake and energy intake:estimated energy requirement

Associations of EF, MF, SF and adiposity measures with EI and EI:EER are presented in Table 3. All measures of EF, MF and SF were strongly and positively associated with both EI and EI:EER in both children and adolescents, with the exception of no association between $\mathrm{MF}_{\text {energy\% }}$ and $\mathrm{EI}$ in children. Although BMI percentile and WC were positively associated with EI in children only, they were inversely associated with EI:EER in both children and adolescents.

\section{Associations of eating frequency, meal frequency and snack frequency with adiposity measures}

Table 4 shows the associations of EF, MF and SF with adiposity measures. After adjustment for potential confounding factors, except for EI:EER (model 1), all measures of EF, MF and SF showed inverse or null associations with BMI percentile and WC in both age groups. However, further adjustment for EI:EER (model 2) resulted in positive associations of $\mathrm{EF}$ with BMI percentile (children only) and WC. For MF, after full adjustment including EI:EER (model 2), there was no association in children, irrespective of the definition of meals, whereas only 
Table 2. Eating frequency (EF), meal frequency and snack frequency according to categories of subjects characteristics: National Health and Nutrition Examination Survey (NHANES) 2003-2012*

(Mean values with their standard errors)

\begin{tabular}{|c|c|c|c|c|c|c|c|c|c|c|c|c|c|c|}
\hline & \multicolumn{7}{|c|}{ Children aged $6-11$ years $(n 4346)$} & \multicolumn{7}{|c|}{ Adolescents aged $12-19$ years ( $n$ 6338) } \\
\hline & \multirow[b]{2}{*}{$n$} & \multicolumn{2}{|c|}{$\begin{array}{c}E F \\
\text { (times/d) } \dagger\end{array}$} & \multicolumn{2}{|c|}{$\begin{array}{c}\mathrm{MF}_{\text {energy\% }} \\
\text { (times/d)†‡ }\end{array}$} & \multicolumn{2}{|c|}{$\begin{array}{c}\mathrm{SF}_{\text {energy\% }} \\
\text { (times/d)†‡ }\end{array}$} & \multirow[b]{2}{*}{$n$} & \multicolumn{2}{|c|}{$\begin{array}{c}E F \\
\text { (times/d) } \dagger\end{array}$} & \multicolumn{2}{|c|}{$\begin{array}{c}\mathrm{MF}_{\text {energy\% }} \\
\text { (times/d) †‡ }\end{array}$} & \multicolumn{2}{|c|}{$\begin{array}{c}\mathrm{SF}_{\text {energy\% }} \\
\text { (times/d)†‡ }\end{array}$} \\
\hline & & Mean & $\mathrm{SE}$ & Mean & SE & Mean & $\mathrm{SE}$ & & Mean & SE & Mean & SE & Mean & $\mathrm{SE}$ \\
\hline \multicolumn{15}{|l|}{ Sex } \\
\hline Boys & 2151 & 4.83 & 0.04 & 2.91 & 0.02 & 1.91 & 0.04 & 3240 & 4.20 & 0.04 & 2.68 & 0.02 & 1.53 & 0.04 \\
\hline Girls & 2195 & 4.68 & 0.04 & $2 \cdot 84$ & 0.02 & 1.84 & 0.03 & 3098 & 4.06 & 0.03 & 2.68 & 0.02 & 1.38 & 0.03 \\
\hline$P \S$ & & \multicolumn{2}{|c|}{0.01} & \multicolumn{2}{|c|}{0.005} & \multicolumn{2}{|c|}{0.18} & & \multicolumn{2}{|c|}{0.01} & \multicolumn{2}{|c|}{0.88} & \multicolumn{2}{|c|}{0.006} \\
\hline \multicolumn{15}{|l|}{ Race/ethnicity } \\
\hline Non-Hispanic white & 1275 & 4.84 & 0.04 & $2 \cdot 88$ & 0.02 & 1.96 & 0.04 & 1805 & 4.19 & 0.04 & 2.71 & 0.02 & 1.48 & 0.03 \\
\hline Non-Hispanic black & 1161 & 4.37 & 0.04 & $2 \cdot 86$ & 0.02 & 1.51 & 0.04 & 1965 & 3.86 & 0.05 & 2.58 & 0.02 & $1 \cdot 28$ & 0.04 \\
\hline Mexican-American & 1190 & 4.77 & 0.04 & 2.90 & 0.02 & 1.87 & 0.05 & 1719 & $4 \cdot 10$ & 0.05 & $2 \cdot 67$ & 0.03 & 1.43 & 0.05 \\
\hline Others & 720 & 4.80 & 0.07 & $2 \cdot 88$ & 0.03 & 1.91 & 0.06 & 849 & 4.21 & 0.07 & 2.65 & 0.04 & 1.57 & 0.05 \\
\hline$P \S$ & & \multicolumn{2}{|c|}{$<0.0001$} & \multicolumn{2}{|c|}{0.68} & \multicolumn{2}{|c|}{$<0.0001$} & & \multicolumn{2}{|c|}{$<0.0001$} & \multicolumn{2}{|c|}{0.0008} & \multicolumn{2}{|c|}{0.0001} \\
\hline Family poverty income & & & & & & & & & & & & & & \\
\hline$<130 \%$ & 1911 & 4.68 & 0.04 & $2 \cdot 90$ & 0.02 & 1.78 & 0.05 & 2602 & 4.06 & 0.04 & $2 \cdot 68$ & 0.02 & 1.39 & 0.03 \\
\hline $130-349 \%$ & 1519 & 4.75 & 0.06 & $2 \cdot 85$ & 0.02 & 1.90 & 0.06 & 2287 & 4.07 & 0.04 & $2 \cdot 66$ & 0.02 & 1.41 & 0.04 \\
\hline$\geq 350 \%$ & 916 & 4.85 & 0.05 & 2.89 & 0.02 & 1.97 & 0.05 & 1449 & 4.26 & 0.05 & 2.71 & 0.02 & 1.56 & 0.05 \\
\hline$P \S$ & & & & & & & & & 0.0 & & 0 & & & \\
\hline Physical activity\|l & & & & & & & & & & & & & & \\
\hline Low & 735 & 4.72 & 0.08 & $2 \cdot 88$ & 0.03 & 1.84 & 0.09 & 998 & 3.88 & 0.05 & 2.58 & 0.03 & 1.30 & 0.05 \\
\hline Moderate & 998 & 4.69 & 0.05 & $2 \cdot 84$ & 0.03 & 1.85 & 0.05 & 2568 & 4.06 & 0.03 & 2.69 & 0.02 & 1.36 & 0.03 \\
\hline Active & 2613 & 4.79 & 0.04 & $2 \cdot 89$ & 0.02 & 1.90 & 0.04 & 2772 & 4.29 & 0.04 & 2.70 & 0.02 & 1.58 & 0.04 \\
\hline$P \S$ & & & & & & & & & $<0$ & & 0.0 & & $<0$ & 01 \\
\hline Watching television an & comput & use & & & & & & & & & & & & \\
\hline$<2 \mathrm{~h} / \mathrm{d}$ & 1117 & $4 \cdot 70$ & 0.06 & $2 \cdot 85$ & 0.02 & 1.86 & 0.06 & 1116 & $4 \cdot 21$ & 0.06 & 2.72 & 0.03 & 1.49 & 0.06 \\
\hline$\geq 2$ to $<4 \mathrm{~h} / \mathrm{d}$ & 1824 & 4.78 & 0.05 & 2.92 & 0.02 & 1.86 & 0.05 & 1670 & 4.20 & 0.06 & 2.73 & 0.03 & 1.47 & 0.05 \\
\hline$\geq 4$ to $<6 \mathrm{~h} / \mathrm{d}$ & 864 & 4.78 & 0.06 & 2.83 & 0.03 & 1.95 & 0.05 & 1372 & 4.20 & 0.06 & 2.63 & 0.03 & 1.56 & 0.06 \\
\hline$\geq 6 \mathrm{~h} / \mathrm{d}$ & 541 & 4.75 & 0.09 & 2.87 & 0.03 & 1.88 & 0.09 & 2180 & 4.03 & 0.03 & 2.66 & 0.02 & 1.37 & 0.03 \\
\hline$P \S$ & & & & & & & & & 0. & & 0 & & & \\
\hline Survey cycle & & & & & & & & & & & & & & \\
\hline 2003-2004 & 785 & 4.81 & 0.06 & 2.90 & 0.03 & 1.91 & 0.06 & 1800 & $4 \cdot 23$ & 0.08 & $2 \cdot 67$ & 0.03 & 1.55 & 0.06 \\
\hline 2005-2006 & 894 & 4.75 & 0.06 & $2 \cdot 85$ & 0.02 & 1.90 & 0.06 & 1773 & $4 \cdot 21$ & 0.06 & $2 \cdot 68$ & 0.02 & 1.53 & 0.06 \\
\hline 2007-2008 & 831 & 4.73 & 0.06 & $2 \cdot 88$ & 0.03 & 1.85 & 0.06 & 872 & 4.06 & 0.06 & 2.70 & 0.03 & 1.36 & 0.05 \\
\hline 2009-2010 & 876 & 4.73 & 0.05 & 2.89 & 0.03 & 1.85 & 0.05 & 955 & $4 \cdot 10$ & 0.06 & 2.72 & 0.03 & 1.37 & 0.05 \\
\hline 2011-2012 & 960 & 4.76 & 0.08 & $2 \cdot 86$ & 0.03 & 1.89 & 0.08 & 938 & 4.07 & 0.06 & 2.63 & 0.04 & 1.43 & 0.05 \\
\hline$P \S$ & & & & & & & & & 0 & & 0 & & 0 & \\
\hline
\end{tabular}

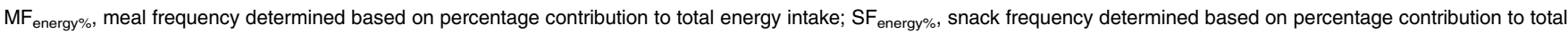
energy intake.

* All mean values are weighted to reflect the survey design characteristics. Analyses are based on subjects with complete data on two 24-h dietary recalls as well as complete information on the variables of interest. All dietary variables are based on average values of the two 24-h dietary recalls.

$\dagger$ On the basis of all occasions except for those providing $<210 \mathrm{~kJ}$ of energy.

$\ddagger$ A meal was defined as any eating episode comprising $\geq 15 \%$ of total energy intake, regardless of the time of the day or composition of foods and beverages consumed; all other eating episodes were classified as snacks.

$\S P$ values for heterogeneity based on Wald's $F$ test.

II The physical activity variable was created as follows. For children aged 6-11 years, the response to the question on the number of times per week that play or exercise was hard enough to induce sweating in the past 7d (NHANES 2009-2012) or without a specified period (NHANES 2003-2008): 0-3 times $=$ low, 4-6 times $=$ moderate and 7 times $=$ active. For adolescents aged $12-19$ years, the responses to two different questions on any leisure time moderate or vigorous activities lasting $\geq 10$ min in the past $30 \mathrm{~d}$ (NHANES 2003-2006) or without a specified period (NHANES 2007-2012): no to both two questions=low, yes to one question=moderate and yes to both two questions $=$ active

$\mathrm{MF}_{\text {energy } \%}$ showed inverse associations in adolescents. After full adjustment (model 2), all measures of SF were positively associated with BMI percentile and WC in children, whereas only $\mathrm{SF}_{\text {energy\% }}$ showed positive associations in adolescents.

\section{Associations of eating frequency, meal frequency and snack frequency with overweight and abdominal obesity}

Associations of EF, MF and SF with overweight and abdominal obesity are shown in Table 5 . When analysed without adjustment for EI:EER (model 1), all measures of EF, MF and
SF showed inverse or null associations in both age groups. However, after adjustment for EI:EER (model 2), EF was positively associated with the risk of overweight (children only) and abdominal obesity. Although all measures of MF showed no associations, all measures of SF were associated with higher risks of overweight and abdominal obesity in children. The associations varied in adolescents, depending on the definition of meals and snacks. Although $\mathrm{MF}_{\text {energy\% }}$ was associated with a lower risk of overweight, $\mathrm{SF}_{\text {energy\% }}$ and $\mathrm{MF}$ based on self-report showed positive associations with both overweight and abdominal obesity. 


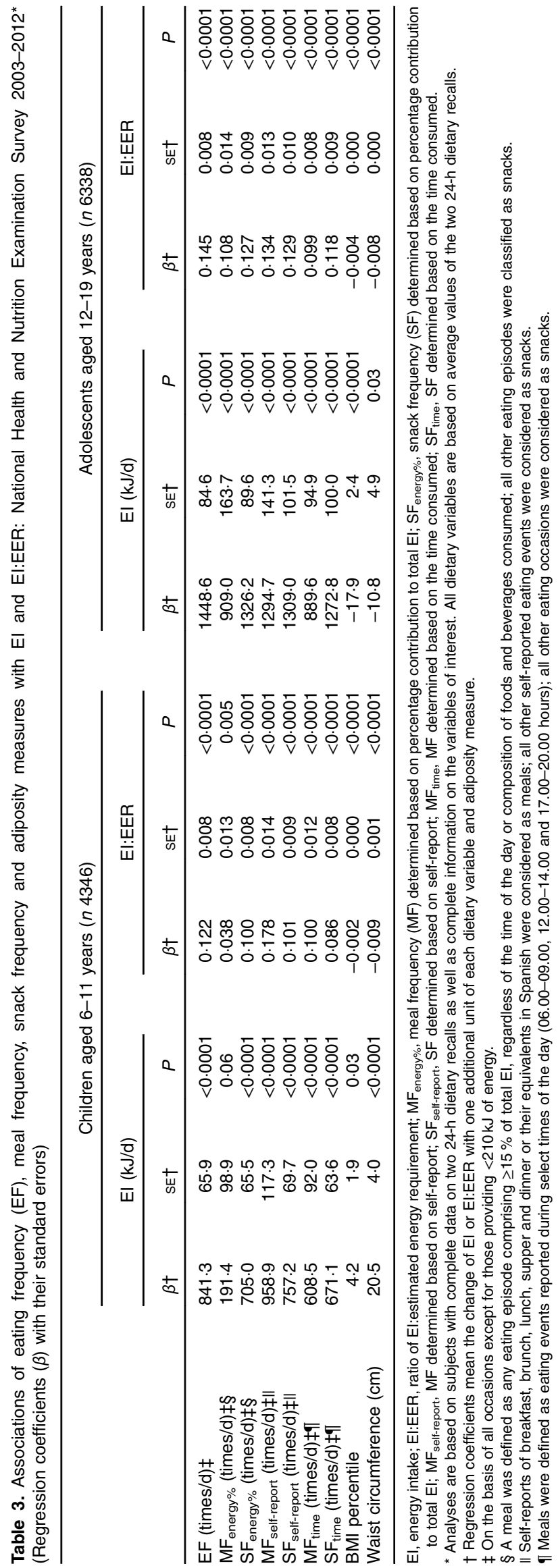

\section{Discussion}

To the authors' knowledge, this is the first study to examine associations of different measures of $\mathrm{EF}, \mathrm{MF}$ and $\mathrm{SF}$ with adiposity measures in a representative sample of US children and adolescents. In the multivariate analyses without taking into account EI misreporting (assessed by EI:EER), all measures of $\mathrm{EF}, \mathrm{MF}$ and SF showed inverse or null associations in both children and adolescents. However, after full adjustment including EI:EER, a completely different picture emerged, showing positive associations between EF and overweight (children only) and abdominal obesity. All SF variables were also associated with higher risks of overweight and abdominal obesity in children, with no associations for all MF variables. The associations in adolescents were inconsistent, which depended on the definition of meals and snacks. Thus, adjustment for the misreporting of EI, as well as the definition of MF and $\mathrm{SF}$, radically changed the results of the present analysis.

Well-designed previous studies on the association between $\mathrm{EF}$ and measures of body fatness have shown mixed findings. In a 10-year prospective study of girls, less-frequent eating at baseline (9-10 years of age) predicted a greater gain in BMI and $\mathrm{WC}^{(7)}$. Conversely, another prospective study of girls aged 8-12 years showed that higher $\mathrm{EF}$ ( $\geq 6$ times/d), compared with moderate EF ( $\geq 4$ to $<6$ times/d), was associated with higher increase in BMI $z$-score between 8-12 and 11-19 years of age ${ }^{(21)}$. In addition, there was a cross-sectional association between $\mathrm{EF}$ and BMI $z$-score and waist:height ratio in centrally obese children aged 9-10 years, after adjustment for EI misreporting ${ }^{(22)}$. In a cross-sectional analysis where only plausible EI reporters were included, there was no association between EF and BMI percentile in all three groups of young people (3-5, 6-11 and 12-19 years) ${ }^{(25)}$. A positive association between EF and BMI $z$-score emerged in adolescents aged 11-18 years (but not in children aged $4-10$ years) after adjustment for EI misreporting ${ }^{(26)}$. In the present study, positive associations between EF and adiposity measures were shown after adjustment for EI:EER, which is consistent with several previous studies ${ }^{(21-26)}$. To the authors' knowledge, there has been no attempt to investigate the effects of MF and SF separately in young populations; however, one cross-sectional study in US adults ${ }^{(55)}$ has shown that after excluding implausible EI reporters SF, but not MF, as defined mainly based on self-report and energy content, was positively associated with BMI, which is consistent with the present observations in children, but not in adolescents. These discrepant findings may be, at least partly, explained by differences in the characteristics and lifestyles of the populations, definitions of $\mathrm{EF}, \mathrm{MF}$ and $\mathrm{SF}$, dietary assessment methods, adiposity measures and potential confounding factors considered, in addition to under-reporting of EF, MF and SF by obese or overweight subjects.

The main finding of the present study that EF was positively associated with adiposity measures seems plausible given the observed positive association of EF with EI. An increased EF may contribute to an increased EI, resulting in fat mass deposition. Although all measures of MF and SF were positively associated with EI, only SF was associated with adiposity 
Table 4. Associations of eating frequency (EF), meal frequency and snack frequency with adiposity measures: National Health and Nutrition Examination Survey 2003-2012* (Regression coefficients $(\beta)$ with their standard errors)

\begin{tabular}{|c|c|c|c|c|c|c|c|c|c|c|c|c|}
\hline & \multicolumn{6}{|c|}{ Children aged $6-11$ years ( $n$ 4346) } & \multicolumn{6}{|c|}{ Adolescents aged $12-19$ years ( $n$ 6338) } \\
\hline & \multicolumn{3}{|c|}{ Model $1 \dagger$} & \multicolumn{3}{|c|}{ Model 2‡ } & \multicolumn{3}{|c|}{ Model 1† } & \multicolumn{3}{|c|}{ Model 2‡ } \\
\hline & $\beta \S$ & $\mathrm{SE} \S$ & $P$ & $\beta \S$ & $\mathrm{SE} \S$ & $P$ & $\beta \S$ & $\mathrm{SE} \S$ & $P$ & $\beta \S$ & $\mathrm{SE} \S$ & $P$ \\
\hline \multicolumn{13}{|l|}{ EF (times/d)\| } \\
\hline BMI percentile & -1.31 & 0.69 & 0.06 & 2.09 & 0.79 & 0.01 & -4.53 & 0.51 & $<0.0001$ & 0.39 & 0.59 & 0.51 \\
\hline Waist circumference (cm) & -0.28 & 0.20 & 0.17 & 1.20 & 0.25 & $<0.0001$ & -1.76 & 0.27 & $<0.0001$ & 0.79 & 0.30 & 0.01 \\
\hline \multicolumn{13}{|l|}{$\mathrm{MF}_{\text {energy\% }}($ times/d)\|ा } \\
\hline BMI percentile & $-2 \cdot 30$ & 1.12 & 0.04 & -1.56 & $1 \cdot 18$ & 0.19 & -7.88 & $1 \cdot 12$ & $<0.0001$ & -4.42 & 1.02 & $<0.0001$ \\
\hline Waist circumference (cm) & -0.38 & 0.37 & 0.31 & -0.07 & 0.40 & 0.87 & -2.93 & 0.47 & $<0.0001$ & $-1 \cdot 20$ & 0.46 & 0.01 \\
\hline \multicolumn{13}{|l|}{$\mathrm{SF}_{\text {energy\% }}($ times $/ \mathrm{d}) \| \boldsymbol{\Phi}$} \\
\hline BMI percentile & -0.62 & 0.65 & 0.34 & 2.08 & 0.72 & 0.005 & -2.49 & 0.59 & $<0.0001$ & 1.88 & 0.64 & 0.004 \\
\hline Waist circumference (cm) & -0.15 & 0.20 & 0.44 & 0.99 & 0.23 & $<0.0001$ & -1.01 & 0.33 & 0.003 & 1.17 & 0.33 & 0.0006 \\
\hline \multicolumn{13}{|l|}{$\mathrm{MF}_{\text {self-report }}\left(\right.$ times $/ \mathrm{d}$ ) $\|^{\star \star *}$} \\
\hline BMI percentile & $-7 \cdot 36$ & 1.58 & $<0.0001$ & -2.95 & 1.55 & 0.06 & -4.84 & 0.92 & $<0.0001$ & 0.51 & 0.97 & 0.60 \\
\hline Waist circumference $(\mathrm{cm})$ & -2.05 & 0.52 & 0.0002 & -0.13 & 0.55 & 0.81 & -1.77 & 0.46 & 0.0002 & 0.90 & 0.46 & 0.052 \\
\hline \multicolumn{13}{|l|}{$\mathrm{SF}_{\text {self-report }}($ times $/ \mathrm{d}) \|^{\star *}$} \\
\hline BMI percentile & 0.05 & 0.81 & 0.95 & $2 \cdot 82$ & 0.88 & 0.002 & -3.89 & 0.57 & $<0.0001$ & 0.24 & 0.60 & 0.70 \\
\hline Waist circumference $(\mathrm{cm})$ & 0.12 & 0.24 & 0.63 & 1.28 & 0.27 & $<0.0001$ & -1.55 & 0.29 & $<0.0001$ & 0.53 & 0.30 & 0.09 \\
\hline \multicolumn{13}{|l|}{$\mathrm{MF}_{\text {time }}($ times/d)\|†† } \\
\hline BMI percentile & -2.50 & 0.87 & 0.005 & -0.02 & 0.86 & 0.98 & -3.80 & 0.76 & $<0.0001$ & 0.38 & 0.75 & 0.62 \\
\hline Waist circumference $(\mathrm{cm})$ & -0.87 & 0.22 & 0.0002 & 0.17 & 0.24 & 0.49 & $-1 \cdot 28$ & 0.37 & 0.0008 & 0.44 & 0.36 & 0.23 \\
\hline \multicolumn{13}{|l|}{$\mathrm{SF}_{\text {time }}($ times $/ \mathrm{d}) \| \dagger \dagger$} \\
\hline BMI percentile & 0.40 & 0.87 & 0.65 & 2.52 & 0.90 & 0.006 & $-2 \cdot 88$ & 0.74 & 0.0002 & 0.82 & 0.78 & 0.30 \\
\hline Waist circumference $(\mathrm{cm})$ & 0.39 & 0.29 & 0.17 & 1.29 & 0.29 & $<0.0001$ & -1.29 & 0.31 & $<0.0001$ & 0.53 & 0.30 & 0.08 \\
\hline
\end{tabular}

$\mathrm{MF}_{\text {energy\%, }}$, meal frequency (MF) determined based on percentage contribution to total energy intake; $\mathrm{SF}_{\text {energy }}$, snack frequency (SF) determined based on percentage contribution to total energy intake; $\mathrm{MF}_{\text {selt-report, }} \mathrm{MF}$ determined based on self-report; $\mathrm{SF}_{\text {self-report, }} \mathrm{SF}$ determined based on self-report; $\mathrm{MF}_{\text {time }}, \mathrm{MF}$ determined based on the time consumed; $\mathrm{SF}_{\text {time, }}, \mathrm{SF}$ determined based on the time consumed.

* Analyses are based on subjects with complete data on two 24-h dietary recalls as well as complete information on the variables of interest. All dietary variables are based on average values of the two 24-h dietary recalls.

$†$ Adjusted for sex (boys or girls), age (years, continuous), race/ethnicity (non-Hispanic white, non-Hispanic black, Mexican-American or others), family poverty income ratio ( $<130,130-349$ or $\geq 350 \%$ ), physical activity (low, moderate or active), watching television and computer use ( $<2, \geq 2$ to $<4$, $\geq 4$ to $<6$ or $\geq 6$ h/d), survey cycle (2003-2004, 2005-2006, 2007-2008, 2009-2010 or 2011-2012), protein intake (percentage of energy, continuous), fat intake (percentage of energy, continuous), total sugar intake (percentage of energy, continuous) and dietary fibre intake (g/4184 kJ, continuous).

‡ Adjusted for variables used in model 1 and ratio of energy intake:estimated energy requirement (continuous).

$\S$ Regression coefficients mean the change of adiposity measures with one additional eating occasion per day.

$\|$ On the basis of all occasions except for those providing $<210 \mathrm{~kJ}$ of energy.

I A meal was defined as any eating episode comprising $\geq 15 \%$ of total energy intake, regardless of the time of the day or composition of foods and beverages consumed; all other eating episodes were classified as snacks.

t† Meals were defined as eating events reported during select times of the day (06.00-09.00, 12.00-14.00 and 17.00-20.00 hours); all other eating occasions were considered as snacks. 
Table 5. Associations of eating frequency (EF), meal frequency and snack frequency with overweight and abdominal obesity: National Health and Nutrition Examination Survey 2003-2012* (Odds ratios and $95 \%$ confidence intervals)

\begin{tabular}{|c|c|c|c|c|c|c|c|c|c|c|c|c|}
\hline & \multicolumn{6}{|c|}{ Children aged $6-11$ years $(n$ 4346) } & \multicolumn{6}{|c|}{ Adolescents aged $12-19$ years ( $n$ 6338) } \\
\hline & \multicolumn{3}{|c|}{ Model $1 \dagger$} & \multicolumn{3}{|c|}{ Model $2 \ddagger$} & \multicolumn{3}{|c|}{ Model 1† } & \multicolumn{3}{|c|}{ Model $2 \ddagger$} \\
\hline & OR§ & $95 \% \mathrm{Cl} \S$ & $P$ & ORß & $95 \% \mathrm{Cl} \S$ & $P$ & OR§ & $95 \% \mathrm{Cl}$ & $P$ & OR§ & $95 \% \mathrm{Cl} \S$ & $P$ \\
\hline \multicolumn{13}{|l|}{ EF (times/d)\| } \\
\hline Overweight & 0.92 & $0.83,1.02$ & 0.10 & 1.23 & $1.09,1.39$ & 0.001 & 0.75 & $0.69,0.81$ & $<0.0001$ & 1.09 & $0.98,1.20$ & 0.11 \\
\hline Abdominal obesity** & 0.92 & $0.82,1.04$ & $0 \cdot 17$ & 1.35 & $1 \cdot 17,1.56$ & $<0.0001$ & 0.75 & $0.68,0.83$ & $<0.0001$ & 1.22 & $1 \cdot 10,1 \cdot 35$ & 0.0003 \\
\hline \multicolumn{13}{|l|}{$\mathrm{MF}_{\text {energy\% }}($ times/d)\|†† } \\
\hline Overweight & 0.90 & $0.77,1.04$ & 0.16 & 0.96 & $0.80,1.15$ & 0.66 & 0.64 & $0.55,0.74$ & $<0.0001$ & 0.83 & $0.70,0.98$ & 0.03 \\
\hline Abdominal obesity** & 1.00 & $0.83,1.22$ & 0.97 & 1.14 & $0.91,1.43$ & 0.27 & 0.71 & $0.59,0.85$ & 0.0002 & 1.02 & $0.83,1.25$ & 0.86 \\
\hline \multicolumn{13}{|l|}{$\mathrm{SF}_{\text {energy\% }}$ (times/d)\|†† } \\
\hline Overweight & 0.95 & $0.86,1.05$ & 0.33 & 1.19 & $1.06,1.34$ & 0.003 & 0.84 & $0.76,0.92$ & 0.0003 & 1.16 & $1.04,1.29$ & 0.006 \\
\hline Abdominal obesity** & 0.93 & $0.83,1.04$ & 0.21 & 1.24 & $1.08,1.42$ & 0.003 & 0.82 & $0.73,0.92$ & 0.0008 & $1 \cdot 21$ & $1.08,1.36$ & 0.001 \\
\hline \multicolumn{13}{|l|}{ 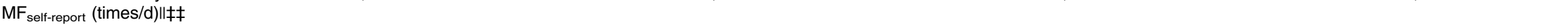 } \\
\hline Overweight & 0.59 & $0.47,0.73$ & $<0.0001$ & 0.83 & $0.65,1.07$ & 0.15 & 0.79 & $0.69,0.91$ & 0.0008 & 1.22 & $1.05,1.43$ & 0.01 \\
\hline Abdominal obesity ${ }^{\star \star}$ & 0.78 & $0.59,1.02$ & 0.07 & 1.34 & $0.99,1.82$ & 0.06 & 0.79 & $0.66,0.95$ & 0.01 & 1.36 & $1 \cdot 11,1 \cdot 67$ & 0.003 \\
\hline \multicolumn{13}{|l|}{ 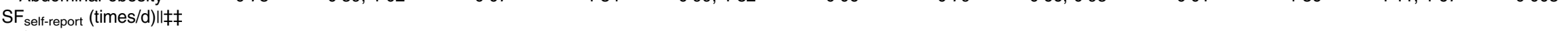 } \\
\hline Overweight & 1.02 & $0.90,1.15$ & 0.75 & 1.29 & $1.12,1.49$ & 0.0005 & 0.76 & $0.69,0.83$ & $<0.0001$ & 1.01 & $0.91,1.12$ & 0.82 \\
\hline Abdominal obesity ${ }^{\star *}$ & 0.97 & $0.85,1 \cdot 10$ & 0.58 & 1.27 & $1.09,1.48$ & 0.002 & 0.76 & $0.68,0.85$ & $<0.0001$ & 1.09 & $0.97,1.23$ & 0.16 \\
\hline \multicolumn{13}{|l|}{$\mathrm{MF}_{\text {time }}($ times $/ \mathrm{d}) \| \S \S$} \\
\hline Overweight & 0.82 & $0.74,0.91$ & 0.0003 & 0.99 & $0.88,1.12$ & 0.91 & 0.84 & $0.75,0.93$ & 0.001 & 1.09 & $0.97,1.21$ & 0.15 \\
\hline \multirow{2}{*}{\multicolumn{13}{|c|}{$\mathrm{SF}_{\text {time }}($ times/d)\|§§ }} \\
\hline & & & & & & & & & & & & \\
\hline Overweight & 1.06 & $0.94,1.19$ & 0.33 & 1.28 & $1 \cdot 12,1.46$ & 0.0002 & 0.79 & $0.71,0.87$ & $<0.0001$ & 1.02 & $0.90,1 \cdot 15$ & 0.74 \\
\hline Abdominal obesity** & 1.08 & $0.94,1.24$ & 0.27 & 1.38 & $1 \cdot 17,1.62$ & 0.0001 & 0.80 & $0.71,0.91$ & 0.0004 & 1.11 & $0.97,1.26$ & 0.14 \\
\hline
\end{tabular}

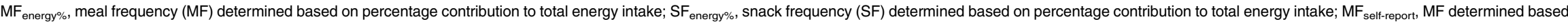
on self-report; $\mathrm{SF}_{\text {sell-report, }} \mathrm{SF}$ determined based on self-report; $\mathrm{MF}_{\text {time, }}, \mathrm{MF}$ determined based on the time consumed; $\mathrm{SF}_{\text {time, }}$, $\mathrm{SF}$ determined based on the time consumed.

* Analyses are based on subjects with complete data on two 24-h dietary recalls as well as complete information on the variables of interest. All dietary variables are based on average values of the two 24-h dietary recalls.

† Adjusted for sex (boys or girls), age (years, continuous), race/ethnicity (non-Hispanic white, non-Hispanic black, Mexican-American or others), family poverty income ratio ( $<130,130-349$ or $\geq 350 \%$ ), physical activity (low, moderate or active), watching television and computer use ( $<2, \geq 2$ to $<4, \geq 4$ to $<6$ or $\geq 6 \mathrm{~h} / \mathrm{d})$, survey cycle (2003-2004, 2005-2006, 2007-2008, 2009-2010 or 2011-2012), protein intake (percentage of energy, continuous), fat intake (percentage of energy, continuous), total sugar intake (percentage of energy, continuous) and dietary fibre intake (g/4184kJ, continuous).

¥ Adjusted for variables used in model 1 and ratio of energy intake:estimated energy requirement (continuous).

$\S$ OR for overweight and abdominal obesity per one increase of eating occasion per day.

$\|$ On the basis of all occasions except for those providing $<210 \mathrm{~kJ}$ of energy.

I Including obesity. Defined as BMI $\geq 85$ th percentle of BMI for age according to growth charts available from the Centers for Disease Control and Prevention ${ }^{(35,36}$

** Defined as waist circumference equal or above the sex- and age-specific 90th percentile based on data from NHANES III (1988-1994) ${ }^{(37)}$.

t† A meal was defined as any eating episode comprising $\geq 15 \%$ of total energy intake, regardless of the time of day or composition of foods and beverages consumed; all other eating episodes were classified as snacks.

$\S \S$ Meals were defined as eating events reported during select times of the day (06.00-09.00, 12.00-14.00 and 17.00-20.00 hours); all other eating occasions were considered as snacks. 
measures in children, which may be due to stronger associations between SF and EF compared with those between $\mathrm{MF}$ and EF. In adolescents, on the other hand, despite consistent positive associations of $\mathrm{MF}$ and SF with EI, the associations of MF and SF with adiposity measures varied depending on the definition of meals and snacks. Given that there is a lack of consensus definition of meals and snacks, the present study has highlighted the wisdom of applying different definitions for meals and snacks in future evaluation of their impact on adiposity status.

In the present study, (the direction of) the association of EF, MF and SF with adiposity measures radically changed after adjustment for EI:EER. Given the positive association of EF, MF and SF with EI:EER and the inverse association of BMI percentile and WC with EI:EER, this may be due to the under-reporting of EF, MF and SF concomitant with the under-reporting of EI by subjects with higher BMI percentile and WC, as also suggested in a previous study in British adults $^{(56)}$. Thus, the present study highlights the key importance of adjusting for EI misreporting in studies of EF, MF and SF in relation to adiposity measures.

The strengths of this study include the use of a variety of published definitions of $\mathrm{EF}, \mathrm{MF}$ and $\mathrm{SF}$ based on detailed dietary information obtained from two 24-h dietary recalls, measured anthropometric data and the use of an individualised measure of EER to assess misreporting of EI in a large representative sample of US children and adolescents. However, there are also several limitations inherent to this study. First, the cross-sectional nature of the present study does not permit the assessment of causality owing to the uncertain temporality of the association, and only a prospective study would provide better understanding of the relationships of $\mathrm{EF}, \mathrm{MF}$ and SF with adiposity measures

In the present study, an eating occasion was classified as any event that provided $\geq 210 \mathrm{~kJ}$ of energy. Although this definition has been used in several previous studies ${ }^{(26,42-45,47)}$, some arbitrary decision (i.e. energy content) is inevitable by nature. In addition, although MF and SF based on self-report have been most widely used, these are subject to inconsistencies due to differences in individual perceptions, and thus would not necessarily have reduced bias ${ }^{(30)}$. In addition, MF and SF based on EI contribution are, by definition, equal to frequencies of larger (not necessarily 'meal') and smaller (not necessarily 'snack') eating episodes, respectively. Furthermore, MF and SF based on time may be problematic, because eating patterns vary according to lifestyle as well as the cultural environment ${ }^{(30)}$. Thus, results may possibly differ on the basis of other definitions. In any case, as research explicitly examining the impact of these different definitions is limited, it is currently difficult to decide which definition might be most appropriate for meals and snacks. Similar studies using different definitions of meals and snacks need to be carried out before reaching a consensus on what defines meals and snacks.

At present, the only way to obtain unbiased information on energy requirements in free-living settings is to use doubly labelled water ${ }^{(57)}$. This technique is expensive and impractical for application in large-scale epidemiological studies. Instead, in the present study, EER was calculated using equations from the US Dietary Reference Intakes, which have been developed based on a large number of measurements of total energy expenditure by the doubly labelled water method and are highly accurate $\left(R^{2} \geq 0.95\right)^{(48)}$. In the absence of actual, measured total energy expenditure, these equations should serve as the best proxy. Owing to constraints within the data set, the present study did not have a validated and individualised measure of physical activity. Instead, 'low active' level of physical activity was assumed for all subjects for the calculation of EER. This seems adequate for most US children and adolescents, based on accelerometer-based data in NHANES 2003-2006 ${ }^{(49,50)}$. Nevertheless, in very active individuals (e.g. those aged 6-11 years, as has been reported) ${ }^{(50)}$, EER would be underestimated, resulting in an overestimation of EI: EER. Finally, although adjustment for a variety of potential confounding variables was made, residual confounding could not be ruled out.

In conclusion, in this cross-sectional study in a representative sample of US children and adolescents based on NHANES 2003-2012, positive associations between EF and overweight (children only) and abdominal obesity were observed. All measures of SF were associated with higher risks of overweight and abdominal obesity in children, with no associations for all measures of MF. The associations in adolescents were inconsistent, which depended on the definition of meals and snacks. The practical implications of the present study are that higher SF may be a contributing factor to obesity, particularly in children. Nonetheless, over-simplification should be avoided because there is no consensus about what constitutes a snack, a meal or an eating occasion, and results may possibly differ on the basis of other definitions. Further, adjustment for EI:EER radically affected the results of the analysis. This suggests the importance of adjustment for EI:EER, rather than excluding EI misreporters, which may lead to bias. Prospective studies are needed to establish the associations observed in this study.

\section{Acknowledgements}

This study was supported in part by the Grants-in-Aid for Young Scientists (B) from the Ministry of Education, Culture, Sports, Science and Technology of Japan (K. M., grant number 15K16213). The Ministry of Education, Culture, Sports, Science and Technology of Japan had no role in the design, analysis or writing of this article.

K. M. contributed to the concept and design of the study, statistical analysis, data interpretation and manuscript writing. M. B. E. L. critically reviewed the manuscript. All the authors read and approved the final version of the manuscript.

The authors declare that there are no conflicts of interest.

\section{Supplementary material}

For supplementary material/s referred to in this article, please visit http://dx.doi.org/10.1017/S0007114516000854 


\section{References}

1. Ogden CL, Carroll MD, Kit BK, et al. (2012) Prevalence of obesity and trends in body mass index among US children and adolescents, 1999-2010. JAMA 307, 483-490.

2. Ogden CL, Carroll MD, Kit BK, et al. (2014) Prevalence of childhood and adult obesity in the United States, 2011-2012. JAMA 311, 806-814.

3. Williams J, Wake M, Hesketh K, et al. (2005) Health-related quality of life of overweight and obese children. JAMA $\mathbf{2 9 3}$, 70-76.

4. Swallen KC, Reither EN, Haas SA, et al. (2005) Overweight, obesity, and health-related quality of life among adolescents: the National Longitudinal Study of Adolescent Health. Pediatrics 115, 340-347.

5. Berenson GS, Srinivasan SR, Bao W, et al. (1998) Association between multiple cardiovascular risk factors and atherosclerosis in children and young adults. The Bogalusa Heart Study. N Engl J Med 338, 1650-1656.

6. Mahoney LT, Burns TL, Stanford W, et al. (1996) Coronary risk factors measured in childhood and young adult life are associated with coronary artery calcification in young adults: the Muscatine Study. J Am Coll Cardiol 27, 277-284.

7. Ritchie LD (2012) Less frequent eating predicts greater BMI and waist circumference in female adolescents. Am J Clin Nutr 95, 290-296.

8. Keast DR, Nicklas TA \& O'Neil CE (2010) Snacking is associated with reduced risk of overweight and reduced abdominal obesity in adolescents: National Health and Nutrition Examination Survey (NHANES) 1999-2004. Am J Clin Nutr 92, 428-435.

9. Barba G, Troiano E, Russo P, et al. (2006) Total fat, fat distribution and blood pressure according to eating frequency in children living in southern Italy: the ARCA project. Int $J$ Obes (Lond) 30, 1166-1169.

10. Antonogeorgos G, Panagiotakos DB, Papadimitriou A, et al. (2012) Breakfast consumption and meal frequency interaction with childhood obesity. Pediatr Obes 7, 65-72.

11. Eloranta AM, Lindi V, Schwab U, et al. (2012) Dietary factors associated with overweight and body adiposity in Finnish children aged 6-8 years: the PANIC Study. Int I Obes (Lond) 36, 950-955.

12. Franko DL, Striegel-Moore RH, Thompson D, et al. (2008) The relationship between meal frequency and body mass index in black and white adolescent girls: more is less. Int $J$ Obes (Lond) 32, 23-29.

13. Cassimos D, Sidiropoulos H, Batzios S, et al. (2011) Sociodemographic and dietary risk factors for excess weight in a Greek pediatric population living in Kavala, Northern Greece. Nutr Clin Pract 26, 186-191.

14. Mota J, Fidalgo F, Silva R, et al. (2008) Relationships between physical activity, obesity and meal frequency in adolescents. Ann Hum Biol 35, 1-10.

15. Lagiou A \& Parava M (2008) Correlates of childhood obesity in Athens, Greece. Public Health Nutr 11, 940-945.

16. Kosti RI, Panagiotakos DB, Mihas CC, et al. (2007) Dietary habits, physical activity and prevalence of overweight/obesity among adolescents in Greece: the Vyronas study. Med Sci Monit 13, CR437-CR444.

17. Neutzling MB, Taddei JA \& Gigante DP (2003) Risk factors of obesity among Brazilian adolescents: a case-control study. Public Health Nutr 6, 743-749.

18. Lioret S, Touvier M, Lafay L, et al. (2008) Are eating occasions and their energy content related to child overweight and socioeconomic status? Obesity (Silver Spring) 16, $2518-2523$.
19. Toschke AM, Kuchenhoff H, Koletzko B, et al. (2005) Meal frequency and childhood obesity. Obes Res 13, 1932-1938.

20. Kontogianni MD, Farmaki AE, Vidra N, et al. (2010) Associations between lifestyle patterns and body mass index in a sample of Greek children and adolescents. J Am Diet Assoc 110, 215-221.

21. Thompson OM, Ballew C, Resnicow K, et al. (2006) Dietary pattern as a predictor of change in BMI z-score among girls. Int J Obes 30, 176-182.

22. Jennings A, Cassidy A, van Sluijs EM, et al. (2012) Associations between eating frequency, adiposity, diet, and activity in 9-10 year old healthy-weight and centrally obese children. Obesity (Silver Spring) 20, 1462-1468.

23. Ferreira RJ \& Marques-Vidal PM (2008) Prevalence and determinants of obesity in children in public schools of Sintra, Portugal. Obesity (Silver Spring) 16, 497-500.

24. Nicklas TA, Yang SJ, Baranowski T, et al. (2003) Eating patterns and obesity in children. The Bogalusa Heart Study. Am J Prev Med 25, 9-16.

25. Huang TT, Howarth NC, Lin BH, et al. (2004) Energy intake and meal portions: associations with BMI percentile in U.S. children. Obes Res 12, 1875-1885.

26. Murakami K \& Livingstone MBE (2014) Associations of eating frequency with adiposity measures, blood lipid profiles and blood pressure in British children and adolescents. Br J Nutr 111, 2176-2183.

27. McCrory MA, Howarth NC, Roberts SB, et al. (2011) Eating frequency and energy regulation in free-living adults consuming self-selected diets. J Nutr 141, 148S-153S.

28. Bellisle F, McDevitt R \& Prentice AM (1997) Meal frequency and energy balance. BrJ Nutr 77, Suppl. 1, S57-S70.

29. Chapelot D (2011) The role of snacking in energy balance: a biobehavioral approach. J Nutr 141, 158-162.

30. Johnson GH \& Anderson GH (2010) Snacking definitions: impact on interpretation of the literature and dietary recommendations. Crit Rev Food Sci Nutr 50, 848-871.

31. Zipf G, Chiappa M, Porter KS, et al. (2013) National health and nutrition examination survey: plan and operations, 1999-2010. Vital Health Stat 1, 1-37.

32. Johnson CL, Paulose-Ram R, Ogden CL, et al. (2013) National health and nutrition examination survey: analytic guidelines, 1999-2010. Vital Health Stat 2, 1-24.

33. Centers for Disease Control and Prevention \& National Center for Health Statistics (2013) National Health and Nutrition Examination Survey. NHANES response rates and population totals. http://www.cdc.gov/nchs/nhanes/response_rates_cps. htm (accessed December 2014).

34. Centers for Disease Control and Prevention \& National Center for Health Statistics (2014) National Health and Nutrition Examination Survey. Questionnaires, datasets, and related documentation. http://www.cdc.gov/nchs/nhanes/ nhanes_questionnaires.htm (accessed December 2014).

35. Kuczmarski RJ, Ogden CL, Guo SS, et al. (2002) 2000 CDC Growth Charts for the United States: methods and development. Vital Health Stat 11, 1-190.

36. Centers for Disease Control and Prevention \& National Center for Health Statistics (2014) A SAS program for the 2000 CDC Growth Charts (ages 0 to $<20$ years). http://www.cdc.gov/ nccdphp/dnpao/growthcharts/resources/sas.htm (accessed December 2014).

37. Li C, Ford ES, Mokdad AH, et al. (2006) Recent trends in waist circumference and waist-height ratio among US children and adolescents. Pediatrics 118, e1390-e1398.

38. Moshfegh AJ, Rhodes DG, Baer DJ, et al. (2008) The US Department of Agriculture Automated Multiple-Pass Method 
reduces bias in the collection of energy intakes. Am J Clin Nutr 88, 324-332.

39. Blanton CA, Moshfegh AJ, Baer DJ, et al. (2006) The USDA automated multiple-pass method accurately estimates group total energy and nutrient intake. J Nutr 136, 2594-2599.

40. Conway JM, Ingwersen LA, Vinyard BT, et al. (2003) Effectiveness of the US department of agriculture 5-step multiple-pass method in assessing food intake in obese and nonobese women. Am J Clin Nutr 77, 1171-1178.

41. Conway JM, Ingwersen LA \& Moshfegh AJ (2004) Accuracy of dietary recall using the USDA five-step multiple-pass method in men: an observational validation study. I Am Diet Assoc 104, 595-603.

42. Hartline-Grafton HL, Rose D, Johnson CC, et al. (2010) The influence of weekday eating patterns on energy intake and BMI among female elementary school personnel. Obesity (Silver Spring) 18, 736-742.

43. Mills JP, Perry CD \& Reicks M (2011) Eating frequency is associated with energy intake but not obesity in midlife women. Obesity (Silver Spring) 19, 552-559.

44. Yannakoulia M, Melistas L, Solomou E, et al. (2007) Association of eating frequency with body fatness in pre- and postmenopausal women. Obesity (Silver Spring) 15, 100-106.

45. Ma Y, Bertone ER, Stanek EJ 3rd, et al. (2003) Association between eating patterns and obesity in a free-living US adult population. Am J Epidemiol 158, 85-92.

46. Kant AK \& Graubard BI (2015) 40-Year trends in meal and snack eating behaviors of American adults. J Acad Nutr Diet 115, 50-63.

47. Ruidavets JB, Bongard V, Bataille V, et al. (2002) Eating frequency and body fatness in middle-aged men. Int J Obes Relat Metab Disord 26, 1476-1483.
48. Institute of Medicine (2002) Dietary Reference Intakes for Energy, Carbohydrate, Fiber, Fat, Fatty Acids, Cholesterol, Protein and Amino Acids. Washington, DC: National Academies Press.

49. Troiano RP, Berrigan D, Dodd KW, et al. (2008) Physical activity in the United States measured by accelerometer. Med Sci Sports Exerc 40, 181-188.

50. Belcher BR, Berrigan D, Dodd KW, et al. (2010) Physical activity in US youth: effect of race/ethnicity, age, gender, and weight status. Med Sci Sports Exerc 42, 2211-2221.

51. Tooze JA, Kipnis V, Buckman DW, et al. (2010) A mixedeffects model approach for estimating the distribution of usual intake of nutrients: the NCI method. Stat Med 29, $2857-2868$

52. Leech RM, Worsley A, Timperio A, et al. (2015) Characterizing eating patterns: a comparison of eating occasion definitions. Am J Clin Nutr 102, 1229-1237.

53. Murakami K \& Livingstone MBE (2015) Eating frequency is positively associated with overweight and central obesity in US adults. $J$ Nutr 145, 2715-2724.

54. Centers for Disease Control and Prevention \& National Center for Health Statistics (2014) Continuous NHANES web tutorial. http://www.cdc.gov/nchs/tutorials/Nhanes/index_continuous.htm (accessed December 2014).

55. Howarth NC, Huang TTK, Roberts SB, et al. (2007) Eating patterns and dietary composition in relation to BMI in younger and older adults. Int J Obes (Lond) 31, 675-684.

56. Murakami K \& Livingstone MBE (2014) Eating frequency in relation to body mass index and waist circumference in British adults. Int J Obes (Lond) 38, 1200-1206.

57. Livingstone MBE \& Black AE (2003) Markers of the validity of reported energy intake. J Nutr 133, Suppl. 3, 895S-920S. 